\title{
BMJ Open Physical fitness of Latin America children and adolescents: a protocol for a systematic review and meta-analysis
}

\author{
Andrés Godoy-Cumillaf (1) ,' Bruno Bizzozero-Peroni (i) ,2,3 \\ Grant R Tomkinson (D) ,4,5 Javier Brazo-Sayavera (D) ${ }^{6,7}$
}

To cite: Godoy-Cumillaf $A$, Bizzozero-Peroni B,

Tomkinson GR, et al. Physical fitness of Latin America children and adolescents: a protocol for a systematic review and meta-analysis. BMJ Open 2021;11:e047122. doi:10.1136/ bmjopen-2020-047122

- Prepublication history for this paper is available online. To view these files, please visit the journal online ().

Received 19 November 2020 Revised 14 April 2021 Accepted 15 April 2021

\section{A) Check for updates}

(c) Author(s) (or their employer(s)) 2021. Re-use permitted under CC BY-NC. No commercial re-use. See rights and permissions. Published by BMJ.

For numbered affiliations see end of article.

\section{Correspondence to} Professor Andrés GodoyCumillaf;

andres.godoy@uautonoma.cl

\section{ABSTRACT}

Introduction Physical fitness (PF) is an important indicator of health in children and adolescents. Internationally, test batteries have been used to assess overall PF. In Latin America, however, while PF has been widely measured, there is no accepted test battery, making it difficult to monitor and/or compare the PF levels of Latin children. The aim of this study, therefore, is to systematically review and potentially meta-analyse the peer-reviewed literature regarding the assessment of PF in Latin American children and adolescents.

Methods and analysis This systematic review and meta-analysis will follow the Preferred Reporting Items for Systematic Reviews and Meta-Analyses Protocols statement. The systematic literature search will be performed in MEDLINE, Scopus, SciEL0, EMBASE, Cochrane Library, Web of Science, SPORTDiscus, LILACS and Latindex (Spanish) to locate articles published up to April 2021. Eligible studies will include both descriptive and analytic study designs. Meta-analyses are planned for sufficiently homogeneous PF outcomes with regard to statistical and methodological characteristics. Narrative syntheses are planned for PF outcomes that are considered to be too heterogeneous. The statistical program STATA V.15 will be used for meta-analyses, with subgroup analyses performed according to the characteristics of included studies.

Ethics and dissemination This systematic review and meta-analysis protocol is designed to provide updated evidence on the PF of Latin American children and adolescents. Findings from this review may be useful for teachers, researchers and other professionals responsible for paediatric fitness and health promotion/surveillance. The results will be disseminated through peer-reviewed scientific publications, conferences, educational talks and infographics.

PROSPERO registration number CRD42020189892.

\section{INTRODUCTION}

Global guidelines for physical activity (PA) provided by $\mathrm{WHO}^{1}$ recommend that school-age youth accumulate on average 60 min of moderate to vigorous intensity PA on average per day are required to improve their health. ${ }^{2}$ The scientific literature indicates that in addition to meeting PA guidelines, it is important to consider physical

\section{Strengths and limitations of this study}

- This paper describes a systematic review protocol that will be independently performed by two researchers.

- This paper describes the first systematic review on physical fitness for Latin American children and adolescents. It will identify the most commonly used test protocols and will provide descriptive fitness data for Latin American children and adolescents across different age, gender and country groups.

- Study design, sampling and methodological differences may limit comparisons among studies and the generalisability of findings.

fitness (PF) - a proximal outcome of PA levels-as both factors are independently related to health among children and adolescents. $^{3}{ }^{4}$ High childhood $\mathrm{PF}$ is related to better cardiorespiratory and mental health ${ }^{56}$; reduced metabolic risk ${ }^{7}$ and total adiposity ${ }^{8}$; increased bone mineral density ${ }^{78}$; improved coordination and range of movement ${ }^{9}$ and reduced adiposity and better cardiometabolic health later in life. ${ }^{10}$ In addition, both cardiorespiratory and muscular fitness are related to early death and disease in later life. ${ }^{11} 12$ For these reasons, $\mathrm{PF}$ is an important indicator of health in children and adolescents. ${ }^{5}$

Internationally, there are numerous tests and test batteries used to assess PF levels in children and adolescents, ${ }^{13}$ enabling decisionmakers to monitor population health. In this way, various instruments have been developed in different parts of the world in order to report or track population levels of PF, especially for children and adolescents, ${ }^{14-17}$ because schools provide opportunities for population-based testing that do not normally exist for adults. Systematic reviews and metaanalyses have identified and gathered the results of published studies related to $\mathrm{PF}$, which have allowed global comparisons, 
temporal trends and health-related criterion-referenced cut-points to be determined. ${ }^{14-20}$

In Latin America, Brazil developed a fit-for-purpose PF test battery ${ }^{21}$ that has thus far only been used to assess Brazilian youth. In other Latin American countries, PF has been reported using different test protocols, ${ }^{22-27}$ which makes between-country comparisons challenging. Furthermore, international surveillance efforts such as Active Healthy Kids Global Alliance's Global Matrix 3.0 initiative, has identified the paucity of available data, and the need for up-to-date information on the PF levels of Latin American youth. ${ }^{28-35}$

\section{OBJECTIVE}

The aim of this study protocol is to describe a standardised methodology for the development of a systematic review and/or meta-analysis, which describes the available literature on PF for Latin American children and adolescents aged $5-17$ years.

\section{METHODS AND ANALYSIS}

Reporting will follow the Preferred Reporting Items for Systematic Reviews and Meta-Analyses (PRISMA) statement $^{36}$ and the Manual of Collaboration of Cochrane. ${ }^{37}$

\section{Eligibility criteria}

For this protocol, studies retrieved from the peerreviewed literature must have reported on PF and meet the following criteria:

\section{Inclusion criteria}

- Population: Apparently healthy Latin-American school-aged youth aged 5-17 years.

- Exposure: Health-related $\mathrm{PF}^{6}$ components including muscular strength/power, cardiorespiratory endurance and speed.

- Design: Descriptive cross-sectional, cohort, randomised controlled trial, non-randomised experimental study or single-arm pre-post study.

- Outcome: Direct test measures of at least one PF component (eg, cardiorespiratory fitness, muscular fitness, motor fitness), including corresponding summary statistics (eg, means, SDs).

- Period: Published before April 2021.

Exclusion criteria

- Studies evaluating special interest groups of children or adolescents (eg, clinical or athletic groups).

- Studies with a qualitative design.

- Studies that provide duplicate PF data published in another included study.

- Studies reporting self-reported PF measures.

- Other: Published in predatory journals (included in Beall's list of potential predatory journals).

\section{Information sources}

Studies will be identified by searching electronic databases, reference lists, topical systematic analyses/reviews

\begin{tabular}{ll}
\hline Table $1 \quad$ Search strategy for the MEDLINE database \\
\hline Search set Medline & Search set Medline \\
\hline \#1 child* [mh] & \#21 aerobic fitness [tw] \\
\#2 boys [tw] & \#22 aerobic capacity [tw] \\
\hline \#3 girls [tw] & \#23 cardiorespiratory fitness \\
[mh]
\end{tabular}

and personal libraries. The electronic databases will include MEDLINE (via PubMed), Scopus, SciELO, Cochrane Library, LILACS, Web of Science, SPORTDiscus and Latindex (Spanish). Filters will be used to locate studies conducted on humans and published in English, Spanish or Portuguese. Additional studies will be located by supplemental searches of reference lists of included studies, topical systematic reviews and personal libraries. Content experts will be contacted requesting other potentially relevant studies.

\section{Search}

The electronic database search will be limited to keywords, title, and abstract. Search terms will be combined with a Boolean OR and will be searched concurrently with other search groups using the Boolean AND as shown in table 1. Proximity operators ("**) will be used to search for root words. The search strategy will include free-text terms and Medical Subject Heading terms in the case of PubMed.

\section{Patient and public involvement}

No patient involved. 


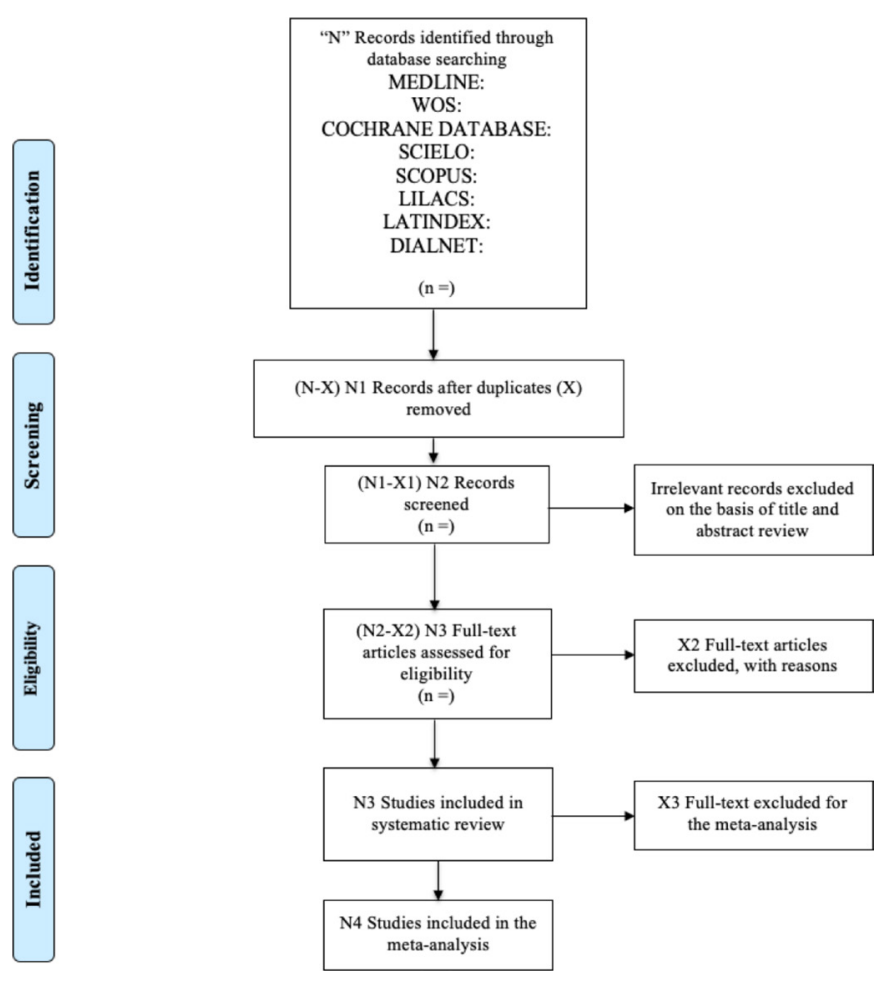

Figure 1 PRISMA flow diagram for identifying, screening and determining the eligibility of and whether to include studies. PRISMA, Preferred Reporting Items for Systematic Reviews and Meta-Analyses.

\section{Study selection}

All database references will be imported into Mendeley Reference Manager (V.1.19.4; Elsevier, London, UK) and deduplicated. Record screening will comprise two levels. Level 1 will involve two researchers independently screening the titles and abstracts against inclusion criteria, with consensus required for further screening. Level 2 will involve two researchers independently screening the full texts against inclusion criteria, with consensus required for final inclusion. If necessary, discrepancies between reviewers will resolved by a third reviewer prior to reaching consensus.

\section{Data collection process}

The process of identification, selection and the inclusion/ exclusion of articles will follow the PRISMA ${ }^{36}$ flow chart (shown in figure 1). Descriptive data will be extracted into a spreadsheet by one researcher using a standardised study-specific template and checked for accuracy by a second researcher. If required, additional information will be requested from the corresponding authors via email (eg, to clarify published results, to request additional data or to avoid double counting data).

\section{Data items}

The following study-specific data will be extracted: (1) name of the first author; (2) year of publication; (3) year of testing; (4) country; (5) study design; (6) calendar age of the participants; (7) biological (maturational) age (if available); (8) sample method/size; (9) PF test; (10) PF test protocol and (11) PF-specific summary statistics. The information will be summarised in a sex-specific 'table of characteristics' (table 2).

\section{Risk of bias in individual studies}

Risk of bias will be independently assessed at the studylevel by two researchers using the Joanna Briggs Institute critical appraisal checklist for analytical cross-sectional studies. ${ }^{38}$ Any disagreements respect to the risk of bias assessment that arise between the reviewers will be resolved through discussion or with a third reviewer. The level of agreement between the reviewers will be reported by calculating the kappa statistics.

\section{Summary measures}

Once the main characteristics of the included studies have been extracted, we will determine whether a metaanalysis is possible (eg, PF data may be meta-analysed to determine temporal trends in PF for Latin American children and adolescents). We will use narrative syntheses to describe the results of included studies should the between-study methodological differences (eg, the mix of PF components, tests, protocols) prevent a metaanalysis, or should fewer than four studies addressing the same outcome be identified. When a minimum of four studies addressing the same outcome have been identified, STATA V.15 software will be used to conduct a random-effects meta-analysis to estimate the pooled effects. Heterogeneity (ie, between-study variability) will be assessed using the $\mathrm{I}^{2}$ statistic, with values of $25 \%, 50 \%$ and $75 \%$ used as thresholds for small, moderate and large, respectively. Forest plots may be used to graphically display the results of the different studies with $95 \%$ CIs, and the pooled outcome with $95 \%$ CIs.

\section{Synthesis of results}

Subgroup analyses and/or meta-regression may be performed considering potential main factors causing heterogeneity (eg, sex, age (calendar or maturational), country, study design, PF test measures). Furthermore, the methodological quality of the selected studies will be considered for the analysis of additional subgroups.

\section{Additional analysis}

To assess the robustness of the summary estimates and detect whether any single study represents a large proportion of heterogeneity, sensitivity analyses will be performed by eliminating each included study one by one from the pooled analyses. Publication bias will be assessed formally by Egger's regression test and visually by funnel plot asymmetry.

\section{ETHICS AND DISSEMINATION}

Ethics committee approval and/or informed consent from patients will not be required, as the data will be published as aggregate data. This study may have implications for public health, as it could provide updated evidence on the PF of Latin American children and adolescents. The 
results obtained will be disseminated to the academic public through peer-reviewed publications, conferences or symposia, and to the general public through social networks, educational talks and infographics.

\section{DISCUSSION}

The aim of this study protocol is to describe a standardised methodology to systematically review and potentially meta-analyse the available scientific literature on PF for Latin American children and adolescents. Available international evidence indicates that $\mathrm{PF}$ is positively and significantly related to the current and future health of children and adolescents. ${ }^{2-10}$

Although data on the PF of Latin American children and adolescents are scarce, several studies have developed norm-referenced data. ${ }^{19-24}$ To date, there are no systematic reviews to our knowledge that have answered the following questions specific to Latin American children and adolescents: What are the most common tests used to measure health-related PF? How well does the PF of Latin American children and adolescents compare to their age-matched and sex-matched peers from other regions of the world? What is the magnitude and direction of the temporal trends in PF for Latin American children and adolescents? This study protocol describes a standardised methodology for the development of a systematic review and/or meta-analysis that will be used to synthesise the peer-reviewed scientific literature, potentially guide future $\mathrm{PF}$ efforts in this region and potentially answer the aforementioned questions.

The limitations of this review may include the absence of published literature and/or typical limitations of systematic reviews/meta-analyses (eg, publication bias, information bias, deficiency in statistical analyses, low methodological quality and heterogeneity of the included studies).

In conclusion, the lack of homogenised information on PF for Latin American children and adolescents highlights the need to systematically review and potentially meta-analyse the available scientific literature, which could meaningfully help to identify literature gaps to guide future studies.

\section{Author affiliations}

${ }^{1}$ Grupo de Investigación en Educación Física, Salud y Calidad de Vida, Facultad de Educación, Universidad Autónoma de Chile, Temuco, Chile

${ }^{2}$ Instituto Superior de Educación Física, Universidad de la Republica, Rivera, Uruguay

${ }^{3}$ Health and Social Research Center, Universidad de Castilla-La Mancha, Cuenca, Spain

${ }^{4}$ Department of Education, Health and Behavior Studies, University of North Dakota, Grand Forks, North Dakota, USA

${ }^{5}$ Alliance for Research in Exercise, Nutrition and Activity (ARENA), School of Health Sciences, University of South Australia, Adelaide, South Australia, Australia ${ }^{6}$ Department of Sports and Computer Science, Universidad Pablo de Olavide, Seville, Spain

${ }^{7}$ Grupo de Investigación en Análisis del Rendimiento Humano, Universidad de la República, Rivera, Uruguay

Twitter Javier Brazo-Sayavera @jbsayavera 
Contributors AG-C, JB-S and BB-P designed the study; $A G-C$ was the principal investigator; JB-S coordinated the study; GT helped with the final design. AG-C wrote the first draft supported by JB-S, BB-P and GT. All the authors reviewed and approved the final version of the manuscript.

Funding The authors have not declared a specific grant for this research from any funding agency in the public, commercial or not-for-profit sectors.

Competing interests None declared.

Patient and public involvement Patients and/or the public were not involved in the design, or conduct, or reporting, or dissemination plans of this research.

Patient consent for publication Not required.

Provenance and peer review Not commissioned; externally peer reviewed.

Open access This is an open access article distributed in accordance with the Creative Commons Attribution Non Commercial (CC BY-NC 4.0) license, which permits others to distribute, remix, adapt, build upon this work non-commercially, and license their derivative works on different terms, provided the original work is properly cited, appropriate credit is given, any changes made indicated, and the use is non-commercial. See: http://creativecommons.org/licenses/by-nc/4.0/.

\section{ORCID iDs}

Andrés Godoy-Cumillaf http://orcid.org/0000-0001-8613-6838

Bruno Bizzozero-Peroni http://orcid.org/0000-0003-0614-5561

Grant R Tomkinson http://orcid.org/0000-0001-7601-9670

Javier Brazo-Sayavera http://orcid.org/0000-0001-6249-5131

\section{REFERENCES}

1 World Health Organization. Global recommendations on physical activity for health. Geneva, Switzerland: WHO, 2010.

2 Bull FC, Al-Ansari SS, Biddle S, et al. World Health Organization 2020 guidelines on physical activity and sedentary behaviour. $\mathrm{Br} J$ Sports Med 2020;54:1451-62.

3 Martínez-Vizcaíno V, Sánchez-López M. [Relationship between physical activity and physical fitness in children and adolescents]. Rev Esp Cardiol 2008;61:108-11.

4 Smith JJ, Eather N, Weaver RG, et al. Behavioral correlates of muscular fitness in children and adolescents: a systematic review. Sports Med 2019;49:887-904.

5 Ruiz JR, Castro-Piñero J, Artero EG, et al. Predictive validity of health-related fitness in youth: a systematic review. Br J Sports Med 2009:43:909-23.

6 Ortega FB, Ruiz JR, Castillo MJ, et al. Physical fitness in childhood and adolescence: a powerful marker of health. Int $J$ Obes 2008;32:1-11.

7 Gomes TN, Dos Santos FK, Katzmarzyk PT, et al. Active and strong: physical activity, muscular strength, and metabolic risk in children. Am J Hum Biol 2017;29:e22904-8.

8 Castillo-Garzón MJ, Ruiz JR, Ortega FB, et al. A Mediterranean diet is not enough for health: physical fitness is an important additional contributor to health for the adults of tomorrow. World Rev Nutr Diet 2007;97:114-38.

9 Lopes L, Póvoas S, Mota J, et al. Flexibility is associated with motor competence in schoolchildren. Scand J Med Sci Sports 2017;27:1806-13.

10 García-Hermoso A, Ramírez-Campillo R, Izquierdo M. Is muscular fitness associated with future health benefits in children and adolescents? A systematic review and meta-analysis of longitudinal studies. Sports Med 2019;49:1079-94.

11 Högström G, Nordström A, Nordström P. Aerobic fitness in late adolescence and the risk of early death: a prospective cohort study of 1.3 million Swedish men. Int J Epidemiol 2016;45:1159-68.

12 Ortega FB, Silventoinen K, Tynelius P, et al. Muscular strength in male adolescents and premature death: cohort study of one million participants. BMJ 2012;345:e7279.

13 Castro-Piñero J, Artero EG, España-Romero V, et al. Criterion-related validity of field-based fitness tests in youth: a systematic review. $\mathrm{Br} \mathrm{J}$ Sports Med 2010;44:934-43.

14 Tomkinson GR, Lang JJ, Tremblay MS, et al. International normative 20 m shuttle run values from 1142026 children and youth representing 50 countries. Br J Sports Med 2017;51:1545-54.

15 Tomkinson GR, Kaster T, Dooley FL, et al. Temporal trends in the standing broad jump performance of 10,940,801 children and adolescents between 1960 and 2017. Sports Med 2021;51:531-48.
16 Tomkinson GR, Lang JJ, Tremblay MS. Temporal trends in the cardiorespiratory fitness of children and adolescents representing 19 high-income and upper middle-income countries between 1981 and 2014. Br J Sports Med 2019;53:478-86.

17 Kolimechkov S. Physical fitness assessment in children and adolescents : A systematic review. Eur J Phys Educ Sport Sci 2017:3:65-79.

18 Lang JJ, Tremblay MS, Ortega FB, et al. Review of criterionreferenced standards for cardiorespiratory fitness: what percentage of 1142026 international children and youth are apparently healthy? Br J Sports Med 2019;53:953-8.

19 Ruiz JR, Cavero-Redondo I, Ortega FB, et al. Cardiorespiratory fitness cut points to avoid cardiovascular disease risk in children and adolescents; what level of fitness should raise a red flag? A systematic review and meta-analysis. Br J Sports Med 2016;50:1451-8.

20 Tomkinson GR, Carver KD, Atkinson F, et al. European normative values for physical fitness in children and adolescents aged 9-17 years: results from 2779165 Eurofit performances representing 30 countries. Br J Sports Med 2018;52:1445-56.

21 Gaya A, Gaya A. Manual do Projeto Esporte Brasil PROESP-Br. Manual de testes e avaliação. UFRGS, 2016.

22 Hernández Mosqueira CM, Fernandes Da Silva S, Fernandes Filho J. Tablas de referencia de condición física en niñas de 10 a 14 años de Chillan, Chile. Revista de Salud Pública 2015;17:667-76.

23 Ramos-Sepúlveda JA, Ramírez-Vélez R, Correa-Bautista JE, et al. Physical fitness and anthropometric normative values among Colombian-Indian schoolchildren. BMC Public Health 2016:16:962-77.

24 Hernández C, Hernández D. Caniuqueo D, et al. Tablas de referencia para aspectos antropométricos y de condición física en estudiantes varones chilenos de 10 a 14 años. Nutrición Hospitalaria 2016;33:1379-84.

25 García-Hermoso A, Cofré-Bolados C, Andrade-Schnettler R, et al. Normative reference values for handgrip strength in Chilean children at 8-12 years old using the empirical distribution and the lambda, mu, and sigma statistical methods. J Strength Cond Res 2021;35:260-6

26 Prieto-Benavides DH, García-Hermoso A, Izquierdo M, et al. Cardiorespiratory fitness Cut-Points are related to body adiposity parameters in Latin American adolescents. Medicina 2019;55:508.

27 Godoy-Cumillaf A, Bruneau-Chávez J, Fuentes-Merino P, et al. Reference values for fitness level and gross motor skills of 4-6-YearOld Chilean children. Int J Environ Res Public Health 2020;17:197.

28 Silva DAS, Christofaro DGD, Ferrari GLdeM, et al. Results from Brazil's 2018 report card on physical activity for children and youth. $J$ Phys Act Health 2018;15:S323-5.

29 Aguilar-Farias N, Miranda-Márquez S, Sadarangani KP, et al. Results from Chile's 2018 report card on physical activity for children and youth. J Phys Act Health 2018;15:S331-2.

30 Andrade S, Ochoa-Avilés A, Freire W, et al. Results from Ecuador's 2018 report card on physical activity for children and youth. J Phys Act Health 2018:15:S344-6.

31 González SA, Triana CA, Abaunza C, et al. Results from Colombia's 2018 report card on physical activity for children and youth. J Phys Act Health 2018:15:S335-7.

32 Galaviz KI, Garcia GA, Gaytán-González A, et al. Results from Mexico's 2018 report card on physical activity for children and youth. $J$ Phys Act Health 2018;15:S384-5.

33 Brazo-Sayavera J, Del Campo C, Rodríguez MJ, et al. Results from Uruguay's 2018 report card on physical activity for children and youth. J Phys Act Health 2018;15:S425-6.

34 Herrera-Cuenca M, Méndez-Pérez B, Landaeta-Jiménez M, et al. Results from Venezuela's 2018 report card on physical activity for children and youth. J Phys Act Health 2018;15:S427-9.

35 Godoy-Cumillaf A, Fuentes-Merino P, Díaz-González A, et al. The effects of physical activity and diet interventions on body mass index in Latin American children and adolescents: a systematic review and meta-analysis. Nutrients 2020;12:1378.

36 Moher D, Shamseer L, Clarke M, et al. Preferred reporting items for systematic review and meta-analysis protocols (PRISMA-P) 2015 statement. Syst Rev 2015;4:1.

37 Higgins J, Green S. Cochrane handbook for systematic review of interventions. Version 5.1.0. [updated March 2011]. The Cochrane Collaboration, 2011. Available: www.handbook.cochrane.org [Accessed 15 Sep 2020].

38 Ma L-L, Wang Y-Y, Yang Z-H. Methodological quality (risk of bias) assessment tools for primary and secondary medical studies: what are they and which is better? Military Med Res 2020;7:7. 